\title{
Strong $\mathrm{CH}^{+} \mathrm{J}=1-0$ emission and absorption in DR21
}

\author{
E. Falgarone ${ }^{1}$, V. Ossenkopf ${ }^{2,3}$, M. Gerin ${ }^{1}$, P. Lesaffre ${ }^{1}$, B. Godard ${ }^{4}$, J. Pearson ${ }^{5}$, S. Cabrit ${ }^{6}$, Ch. Joblin ${ }^{7}$, A. O. Benz ${ }^{8}$, \\ F. Boulanger ${ }^{4}$, A. Fuente ${ }^{9}$, R. Güsten ${ }^{10}$, A. Harris ${ }^{11}$, T. Klein ${ }^{10}$, C. Kramer ${ }^{12}$, S. Lord ${ }^{13}$, P. Martin ${ }^{14}$, \\ J. Martin-Pintado ${ }^{15}$, D. Neufeld ${ }^{16}$, T. G. Phillips ${ }^{17}$, M. Röllig ${ }^{2}$, R. Simon ${ }^{2}$, J. Stutzki ${ }^{2}$, F. van der Tak ${ }^{3,18}$, D. Teyssier ${ }^{19}$, \\ H. Yorke ${ }^{5}$, N. Erickson ${ }^{20}$, M. Fich ${ }^{21}$, W. Jellema ${ }^{3}$, A. Marston ${ }^{19}$, C. Risacher ${ }^{3}$, M. Salez ${ }^{6}$, and F. Schmülling ${ }^{2}$ \\ (Affiliations are available in the online edition)
}

Received 31 March 2010 / Accepted 15 May 2010

\section{ABSTRACT}

We report the first detection of the ground-state rotational transition of the methylidyne cation $\mathrm{CH}^{+}$towards the massive star-forming region $\mathrm{DR} 21$ with the HIFI instrument onboard the Herschel satellite. The line profile exhibits a broad emission line, in addition to two deep and broad absorption features associated with the DR 21 molecular ridge and foreground gas. These observations allow us to determine a ${ }^{12} \mathrm{CH}^{+} J=1-0$ line frequency of $v=835137 \pm 3 \mathrm{MHz}$, in good agreement with a recent experimental determination. We estimate the $\mathrm{CH}^{+}$column density to be a few $10^{13} \mathrm{~cm}^{-2}$ in the gas seen in emission, and $>10^{14} \mathrm{~cm}^{-2}$ in the components responsible for the absorption, which is indicative of a high line of sight average abundance $\left[\mathrm{CH}^{+}\right] /[\mathrm{H}]>1.2 \times 10^{-8}$. We show that the $\mathrm{CH}^{+}$column densities agree well with the predictions of state-of-the-art $\mathrm{C}$-shock models in dense UV-illuminated gas for the emission line, and with those of turbulent dissipation models in diffuse gas for the absorption lines.

Key words. astrochemistry - ISM: molecules - ISM: kinematics and dynamics - turbulence

\section{Introduction}

The methylidyne ion $\mathrm{CH}^{+}$was among the first molecules to be detected in the interstellar medium (ISM) (Douglas \& Herzberg 1941). This reactive ion is prevalent in the diffuse ISM with column densities several orders of magnitude above the predictions of UV-driven equilibrium models (see references in Godard et al. 2009). Apart from the strong emission lines of $\mathrm{CH}^{+}$detected in both the envelope of the Red Rectangle (Hobbs et al. 2004) and NGC 7027 (Cernicharo et al. 1997), all other observations are absorption lines detected at visible wavelengths in the spectra of nearby stars. $\mathrm{CH}^{+}$is a light molecule and its rotational lines lie at submillimeter and far infrared wavelengths. The exact frequencies of the rotational transitions of $\mathrm{CH}^{+}$have remained elusive for a long time because of the extreme reactivity of $\mathrm{CH}^{+}$and the difficulty in isolating it in the laboratory (Pearson \& Drouin 2006). Recent laboratory measurements have led to $v=835.137498(20) \mathrm{GHz}$ for the ground-state transition (Amano 2010). Ground-based astronomical detection of ${ }^{12} \mathrm{CH}^{+}(1-0)$ is prevented by its proximity to a strong atmospheric line of water vapor. The ground-state frequency of the isotopologue ${ }^{13} \mathrm{CH}^{+}$, redshifted by $\sim 5 \mathrm{GHz}$, has superior sky transmission. It has been detected at the Caltech Submillimeter Observatory towards several massive star-forming regions of the inner Galaxy (Falgarone et al., in prep.; Falgarone et al. 2005). The ${ }^{12} \mathrm{CH}^{+}$abundances averaged along these long lines-of-sight (los), confirm the high abundances of this species inferred from visible observations in the local ISM, $\left[\mathrm{CH}^{+}\right] /[\mathrm{H}] \sim 8 \times 10^{-9}$ on average. In this paper, we report the detection of the ${ }^{12} \mathrm{CH}^{+} J=1-0$ transition towards the massive star-forming region DR21, presented in Sect. 2. The

* Herschel is an ESA space observatory with science instruments provided by European-led Principal Investigator consortia and with important participation from NASA.
HIFI observations are described in Sect. 3. The results, given in Sect. 4, are compared to models in Sect. 5.

\section{The DR 21 region}

The massive star-forming region DR 21 is located in the Cygnus X complex at an average distance of $1.7 \mathrm{kpc}$, that of the Cyg OB2 stellar association (see reviews by Jakob et al. 2007; Schneider et al. 2010). The DR 21 molecular core is one of the most massive cores in the Galaxy $\left(2.5 \times 10^{4} M_{\odot}\right.$ at the 1 pc-scale) (Kirby 2009). It is located in front of the main DR 21 HII region comprising five compact HII regions and more diffuse ionised gas (Roelfsema et al. 1989; Wilson et al. 1995; Cyganowski et al. 2003).

The star-forming region DR 21 itself is known to host one of the most powerful molecular outflows shining in vibrationally excited $\mathrm{H}_{2}$ (e.g., Garden \& Carlstrom 1992). The outflow source is heavily extinguished by more than $100 \mathrm{mag}$, and its location is not accurately known (Wilson et al. 1995). High velocity HI (up to $90 \mathrm{~km} \mathrm{~s}^{-1}$ ) associated with the molecular outflow has been detected by the VLA (Russell et al. 1992), indicating that the molecular outflow may be driven by this atomic jet. The outflow shows up as broad wings in $\mathrm{CO}$ and $\mathrm{HCO}^{+}$lines (Kirby 2009). At the position of the HIFI beam, the $v=1-0 \mathrm{~S}(1) \mathrm{H}_{2}$ emission line has a similar broad width (Nadeau \& Béland 1988; Cruz-Gonzalez et al. 2007) to that of the $\mathrm{SiO}$ emission (Motte et al. 2007).

Some line profiles imply that there is foreground gas, which is probably associated with the Cygnus X complex (Jakob et al. 2007; Schneider et al. 2010). A foreground component associated with $\mathrm{W} 75 \mathrm{~N}$ shows up as weak emission lines at $v_{\mathrm{LSR}} \sim$ $8 \mathrm{~km} \mathrm{~s}^{-1}$ in low excitation transitions of $\mathrm{CO}$ and $\mathrm{HCO}^{+}$or atomic carbon, and in the atomic oxygen fine-structure line, with 
$N(\mathrm{O})>5 \times 10^{18} \mathrm{~cm}^{-2}$ (Poglitsch et al. 1996). The total hydrogen column density of this foreground material is estimated to be $N(\mathrm{H}) \sim 1.4 \times 10^{22} \mathrm{~cm}^{-2}$ for an elemental abundance ratio $[\mathrm{O}] /[\mathrm{H}]=3.45 \times 10^{-4}$ (Oliveira et al. 2005), in coherence with $N(\mathrm{H})=(1.3 \pm 0.4) \times 10^{22} \mathrm{~cm}^{-2}$ inferred from $\mathrm{K}$ extinction to DR21 (Marshall et al. 2006). This component is also detected in atomic hydrogen, which indicates that there is saturated absorption of between -5 and $18 \mathrm{~km} \mathrm{~s}^{-1}$ (Roberts et al. 1997). Its estimated HI column density, if $T_{\mathrm{s}}=20 \mathrm{~K}$, is $N(\mathrm{HI})>1.5 \times 10^{21} \mathrm{~cm}^{-2}$.

\section{HIFI observations}

All FIR spectra presented here were obtained in the course of performance verification observations with the HIFI instrument (Pilbratt et al. 2010; de Graauw et al. 2010). Since their main goal was to demonstrate the functionality and performance of the different observing modes, the spectra were taken with a large variety of observing strategies. Most observations were only single-point observations towards the central position of the DR21 HII region at RA $=20^{\mathrm{h}} 39^{\mathrm{m}} 01.1^{\mathrm{s}}$, Dec $=$ $42^{\circ} 19^{\prime} 43.0^{\prime \prime}$ (J2000). At the frequencies around $1 \mathrm{THz}$ discussed here, the Herschel beam covers about 20" HPBW (or $0.16 \mathrm{pc}$ at $1.7 \mathrm{kpc}$ ). Here, we only present data from the wideband spectrometer (WBS) that provides a resolution of $1.1 \mathrm{MHz}$, corresponding to $0.2 \mathrm{~km} \mathrm{~s}^{-1}$ (at $1900 \mathrm{GHz}$ ) and $0.4 \mathrm{~km} \mathrm{~s}^{-1}$ (at $835 \mathrm{GHz}$ ).

The $\mathrm{CH}^{+}$spectrum at $835 \mathrm{GHz}$ was obtained in a singlepoint load-chop observing mode without OFF reference. It uses the internal cold load as reference, having the advantage that we can exclude any self-chopping effects in the line profiles. The mode has, however, the disadvantage that the different optical paths towards the sky and the internal calibration load sometimes lead to standing wave differences that are detected as baseline ripples. We subtracted these manually using the HifiFitFringe pipeline tool. The total integration time on the source was $48 \mathrm{~s}$ leading to a noise level of $0.1 \mathrm{~K}$ for the combination of the spectra from both polarisations at the WBS resolution.

The $\mathrm{H}_{2} \mathrm{O} 1_{11}-0_{00}(1113 \mathrm{GHz})$ line was observed in the frame of a spectral scan in a load-chop mode and an on-source integration time of $30 \mathrm{~s}$. The baseline was calibrated on a distant OFF position $\left(20^{\mathrm{h}} 37^{\mathrm{m}} 10^{\mathrm{s}}, 42^{\circ} 37^{\prime} 00^{\prime \prime}\right)$ because the first selected OFF position, closer to the ON, retained some signal (see Fig. 1). The [CII] line was detected in one of the first double-beam switch raster mapping observations in which neither the logic of the mapping mode worked as expected nor the pointing of the instrument was yet known. The instrument pointing was measured afterwards and the map coordinates were corrected by hand to include the correct offsets. A comparison of the integrated [CII] intensities in the shifted map with the MSX $8 \mu \mathrm{m}$ band shows a very good agreement, confirming the accuracy of the correction. The observations were taken on a $7^{\prime \prime}$ raster that included a $14 \mathrm{~s}$ on-source integration time at each point. To obtain a spatial resolution comparable to that of the other two lines, the map was convolved with a Gaussian beam of $20^{\prime \prime}$ at the DR 21 central position.

\section{Results of the line profile analysis}

The HIFI spectra are displayed in Fig. 1 with, for comparison, an IRAM-30 m spectrum of $\mathrm{HCO}^{+}(1-0)$ (Schneider et al. 2010). The line profiles exhibit both complex emission and absorption because of the multiplicity of velocity components and gas physical conditions within the beam. The line integrated areas in emission and absorption are remarkably comparable. This would

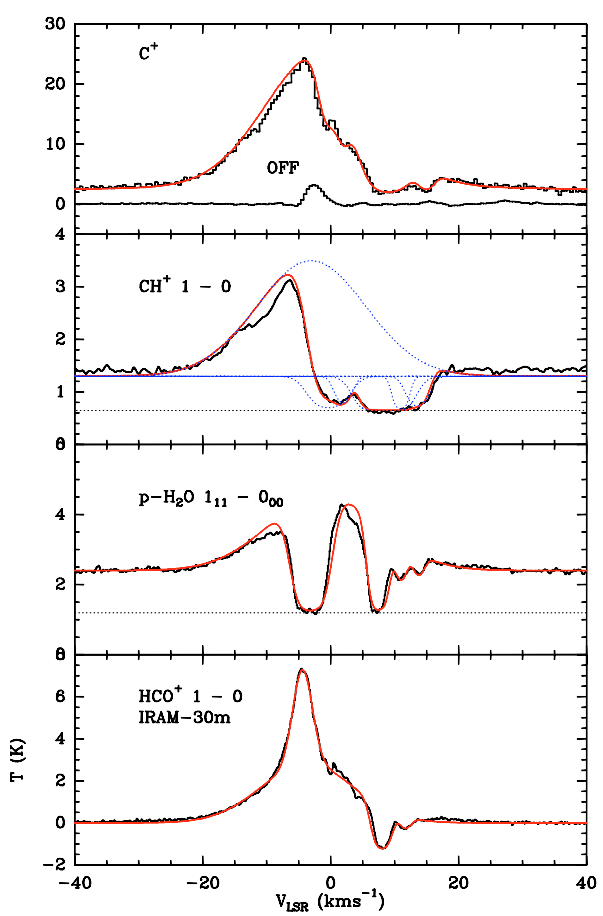

Fig. 1. [CII], $\mathrm{CH}^{+}(1-0)$ and $\mathrm{p}-\mathrm{H}_{2} \mathrm{O}$ Herschel/HIFI spectra (top panels) and $\mathrm{HCO}^{+}(1-0)$ IRAM-30 m spectrum (bottom panel) (Schneider et al. 2010). The HIFI spectra are taken in DSB, hence saturated lines reach to half the continuum level (dotted lines). The weak line in the $\mathrm{C}^{+}$panel is that of an intermediate OFF position. The $\mathrm{CH}^{+}(J=1-0)$ spectrum is shown assuming a rest frequency of $835137 \mathrm{MHz}$. The Gaussian velocity components involved in the empirical model of the $\mathrm{CH}^{+}$profile are shown (dotted blue curves) as well as the resulting models for all lines (red curves) (see Sect. 4).

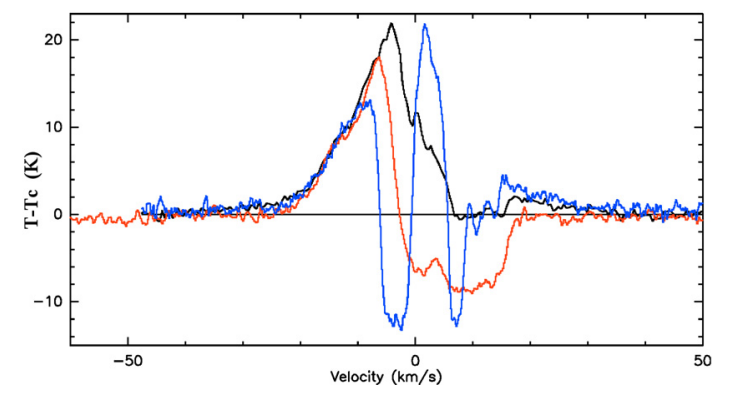

Fig. 2. Superimposition of the $\mathrm{CH}^{+}$(red), $\mathrm{C}^{+}$(black), and $\mathrm{p}-\mathrm{H}_{2} \mathrm{O}$ (blue) spectra (from which the continuum has been subtracted) to illustrate the good coincidence of the blue wing of the line profile when the $\mathrm{CH}^{+}(1-0)$ frequency is $v=835.137 \mathrm{GHz}$. We note also the coincidence of the absorption features in $\mathrm{CH}^{+}(1-0)$ and $\mathrm{C}^{+}$.

lead to a weak or non-detection with low spectral resolution instruments. The dashed lines in the $\mathrm{CH}^{+}$and $\mathrm{p}-\mathrm{H}_{2} \mathrm{O}$ spectra, at about half the continuum level, reveal the broad velocity ranges across which the absorption lines are saturated.

The $\mathrm{CH}^{+}(1-0), \mathrm{C}^{+}$and $\mathrm{p}-\mathrm{H}_{2} \mathrm{O}$ spectra, plotted after their continuum has been subtracted, are superimposed in Fig. 2 for the frequency of the $\mathrm{CH}^{+} J=1-0$ line set to $835.137 \mathrm{GHz}$. We propose in Sect. 5 that the broad line emission originates from a shock associated with the outflow, and we show that the three species, $\mathrm{CH}^{+}, \mathrm{C}^{+}$, and $\mathrm{H}_{2} \mathrm{O}$ are connected with each other in the shock chemistry. The good agreement (within $\pm 1 \mathrm{~km} \mathrm{~s}^{-1}$ ) between the blue wing of the emission line profiles of these three species allows us to adopt $v=835137 \pm 3 \mathrm{MHz}$ as the rest frequency of the ${ }^{12} \mathrm{CH}^{+}(1-0)$ line, which is remarkably consistent with the experimental value measured by Amano (2010). 
Table 1. Empirical models of the broad emission profiles.

\begin{tabular}{llll}
\hline \hline \multirow{2}{*}{ Line } & $v$ & $\Delta v$ & $T$ \\
& $\mathrm{~km} \mathrm{~s}^{-1}$ & $\mathrm{~km} \mathrm{~s}^{-1}$ & $\mathrm{~K}$ \\
\hline $\mathrm{CH}^{+}$ & -3.0 & 20.0 & 2.2 \\
$\mathrm{p}-\mathrm{H}_{2} \mathrm{O}$ & -1.0 & 20.0 & 2.16 \\
$\mathrm{C}^{+}$ & -1.8 & 20.0 & 22 \\
& 0 & 80.0 & 1.0 \\
$\mathrm{HCO}^{+}$ & -3.6 & 17.0 & 2.8 \\
\hline
\end{tabular}

The $\mathrm{CH}^{+} \quad J=1-0$ line profile can be decomposed into a broad emission line and a series of absorption features. We used information from the line profiles of $\mathrm{CO}$ and $\mathrm{HCO}^{+} J=1-0$ (see Fig. 1), the spectra of atomic carbon (Jakob et al. 2007), and other HIFI spectra (Ossenkopf et al. 2010; van der Tak et al. 2010) to constrain the velocity and linewidth of these components. Since the ${ }^{13} \mathrm{CH}^{+}$line has not been observed, the actual $\mathrm{CH}^{+}$emission profile is unknown. We had to rely on the symmetric shapes of the $\mathrm{CO}$ emission lines to model the broad emission wings with Gaussians adjusted on the blue wings of the lines (Table 1 ). Over the velocity range -5 to $17 \mathrm{~km} \mathrm{~s}^{-1}$, not only the continuum level but also the core line emission is absorbed by intervening gas. The broad emission lines $\left(\Delta v=20 \mathrm{~km} \mathrm{~s}^{-1}\right.$, see Table 1) correspond to the emission associated with the outflow shock (see Sect. 5). A still broader component in the [CII] line has a width similar to that of the H66 $\alpha$ recombination lines (Roelfsema et al. 1989). Self-absorption features in the range $\sim-3$ to $2 \mathrm{~km} \mathrm{~s}^{-1}$, caused by the close environment of the DR21 core, are visible in $\mathrm{CO}, \mathrm{HCO}^{+}(4-3)$, and other dense gas tracers (e.g., $\mathrm{NH}_{3}$ inversion lines). The absorption dip at this velocity is more prominent in the $\mathrm{H}_{2} \mathrm{O}\left(1_{1,1}-0_{0,0}\right)$ profile as expected from the high critical density of this transition. We disregarded this velocity range in our analysis because of the unknown line intensity of the dense core emission. In contrast, the absorption at $v>7 \mathrm{~km} \mathrm{~s}^{-1}$ corresponds to the gas that is most likely to be associated with the W75N cloud in the Cygnus $\mathrm{X}$ complex. Its velocity coverage is very similar in the $\mathrm{CH}^{+}$and $\mathrm{C}^{+}$spectra. We fitted three components in this velocity range, guided by the absorption features of the $\mathrm{HCO}^{+} J=1-0$ and $\mathrm{H}_{2} \mathrm{O}$ profiles at $v_{\text {LSR }}=7.5,11$, and $13.7 \mathrm{~km} \mathrm{~s}^{-1}$ shown in Fig. 1, the resulting profiles being in red.

The $\mathrm{CH}^{+}$column density of the emission line was determined by assuming that it is optically thin. This is consistent with the ${ }^{13} \mathrm{CH}^{+}(1-0)$ line not being detected in emission towards galactic massive star-forming regions (Falgarone et al., in prep.; Falgarone et al. 2005). The total $\mathrm{CH}^{+}$column density was therefore assumed to scale with the line integrated area (in $\mathrm{K} \mathrm{km} \mathrm{s}^{-1}$ ) with a coefficient that depends on the excitation temperature. For $40 \mathrm{~K}<T_{\mathrm{ex}}<100 \mathrm{~K}$, the range of $T_{\text {ex }}$ that minimizes $N\left(\mathrm{CH}^{+}\right)$, a lower limit $N_{\mathrm{em}}\left(\mathrm{CH}^{+}\right)=7 \times$ $10^{11} \mathrm{~cm}^{-2} \int T(v) \mathrm{d} v$ was obtained, using the spontaneous decay rate $A_{10}=5.9 \times 10^{-3} \mathrm{~s}^{-1}$ inferred from the dipole moment, $\mu=1.62 \mathrm{D}$ (Kobayashi et al. 1993). In absorption, we inferred the column density from the integral of the optical depth (in $\mathrm{km} \mathrm{s}^{-1}$ ) to be $N_{\mathrm{abs}}\left(\mathrm{CH}^{+}\right)=3.3 \times 10^{12} \mathrm{~cm}^{-2} \int \tau(v) \mathrm{d} v$. It is almost independent of $T_{\text {ex }}$ as long as $T_{\text {ex }} \ll h v / k=39 \mathrm{~K}$, an approximation that is valid for gas of density much lower than the critical density of the transition $\left(>10^{6} \mathrm{~cm}^{-3}\right)$ and not closely associated with intense FIR radiation. The resulting $\mathrm{CH}^{+}$column densities are given in Table 2. Those of the saturated absorption components are lower limits. Although the Gaussian decomposition is by no means unique, it provides an estimate of both the detected column densities and the $\mathrm{CH}^{+}$abundance relative to the total hydrogen $\left[\mathrm{CH}^{+}\right] /[\mathrm{H}]>1.2 \times 10^{-8}$ in the diffuse gas. We
Table 2. $\mathrm{CH}^{+}$Column densities.

\begin{tabular}{|c|c|c|}
\hline Component & $\begin{array}{c}T_{\text {ex }} \\
\mathrm{K}\end{array}$ & $\begin{array}{c}N\left(\mathrm{CH}^{+}\right) \\
\mathrm{cm}^{-2}\end{array}$ \\
\hline Broad line emission & $40 \mathrm{~K}<T_{\mathrm{ex}}<100 \mathrm{~K}$ & $2.2 \times 10^{13}$ \\
\hline Foreground absorption & 3.0 & \\
\hline
\end{tabular}

note that the [CII] line opacity of the foreground gas, averaged over $10 \mathrm{~km} \mathrm{~s}^{-1}$, is $\tau_{\text {[Cii] }}>1.8$. This opacity limit corresponds to a $\mathrm{C}^{+}$column density larger than $2 \times 10^{18} \mathrm{~cm}^{-2}$ for gas of density lower than the critical density of the transition $n_{\mathrm{cr}}\left(\mathrm{C}^{+}\right)$, i.e. $\sim$ a few $10^{3} \mathrm{~cm}^{-3}$, whatever the line excitation temperature (Crawford et al. 1985). The required $\mathrm{C}^{+}$column density is much larger if the transition is thermalized, i.e. $n \gg n_{\mathrm{cr}}\left(\mathrm{C}^{+}\right)$. From the estimated gas column of $1.4 \times 10^{22} \mathrm{~cm}^{-2}$, the expected $\mathrm{C}^{+}$ column is $\sim 2 \times 10^{18} \mathrm{~cm}^{-2}$, assuming a gas-phase carbon abundance of $1.4 \times 10^{-4}$. This value is in close agreement with that derived for the low density limit, strengthening the association of the foreground absorption with gas of densities lower than $\sim$ a few $10^{3} \mathrm{~cm}^{-3}$.

\section{Comparison with model predictions}

The only formation route of the molecular ion $\mathrm{CH}^{+}$is understood to be initiated by the reaction $\mathrm{C}^{+}+\mathrm{H}_{2} \rightarrow \mathrm{CH}^{+}+\mathrm{H}$, which is highly endoenergic $(\Delta E / k=4640 \mathrm{~K})$. The formation of $\mathrm{CH}^{+}$in the cold interstellar medium therefore requires suprathermal energy. Several scenarios have been investigated: C-shocks (Pineau des Forêts et al. 1986), highly illuminated and dense photon-dominated regions (PDR) where $\mathrm{C}^{+}$reacts with vibrationally excited $\mathrm{H}_{2}$ (Sternberg \& Dalgarno 1995; Agundez et al. 2010), turbulent interfaces between the warm and cold neutral medium (Lesaffre et al. 2007), and regions of intermittent turbulent dissipation (TDR models, Godard et al. 2009). In the first and last models, ion-neutral friction plays a major role.

For the DR 21 core, it is unclear which processes are at work in the $\mathrm{CH}^{+}$formation. Broad $\left(\Delta v=20 \mathrm{~km} \mathrm{~s}^{-1}\right)$ emission lines of $\mathrm{SiO}$ (Motte et al. 2007) and vibrationally excited $\mathrm{H}_{2}$ (Cruz-Gonzalez et al. 2007), similar to the broad emission in the HIFI profiles, are observed and suggest the influence of a shock. We now present a preliminary attempt to account for the observed column densities of $\mathrm{CH}^{+}$in this highly irradiated environment.

\section{1. $\mathrm{CH}^{+}$emission line: $\mathrm{C}$-shock models}

We used a steady-state model for C-shocks with time-dependent chemistry and ionization (Flower \& Pineau des Forêts 2003). The chemical network was supplemented with the relevant photo-reactions (Pineau des Forêts, priv. comm.). The $\mathrm{H}_{2}$ and CO self-shielding were assumed to be those of a PDR model at $n_{\mathrm{H}}=10^{4} \mathrm{~cm}^{-3}$. We adopted a pre-shock density $n_{\mathrm{H}}=10^{4} \mathrm{~cm}^{-3}$, a shock velocity $v_{\mathrm{s}}=20 \mathrm{~km} \mathrm{~s}^{-1}$, a magnetic field $B=200 \mu \mathrm{G}$ (from the measure of magnetic field in Roberts et al. 1997), and a standard cosmic-ray ionisation rate of $\zeta=5 \times 10^{-17} \mathrm{~s}^{-1}$. We stopped the computation of the shocks at a neutral flow time of $10^{4} \mathrm{yr}$, which corresponds to the estimated dynamical age of the outflow (lobes of $0.5 \mathrm{pc}$ for a jet velocity up to $100 \mathrm{~km} \mathrm{~s}^{-1}$ ). The $\mathrm{CH}^{+}$column-density was found to have already reached a plateau at this stage in the shock, so the $\mathrm{CH}^{+}$results are insensitive to that choice of age.

Although the incident radiation field is estimated to be $\chi=$ $10^{5}$ in ISRF units (Ossenkopf et al. 2010) for the material closest to the HII region, we keep this value as a free parameter. Figure 3 displays the total column densities of $\mathrm{CH}^{+}$and $\mathrm{H}_{2} \mathrm{O}$ as a 


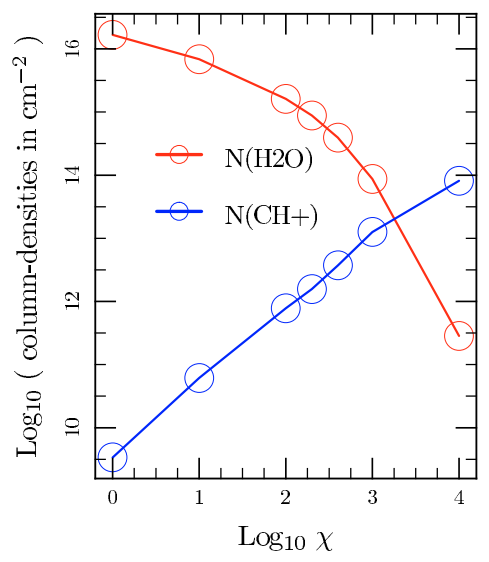

Fig. 3. $\mathrm{CH}^{+}$(blue) and $\mathrm{H}_{2} \mathrm{O}$ (red) column densities integrated through the C-shock model (see text) for a broad range of UV-field irradiations $\chi$. The shielding of the shocked material is $A_{\mathrm{v}}=1$. We note the opposite behaviours of $\mathrm{CH}^{+}$and $\mathrm{H}_{2} \mathrm{O}$ with $\chi$.

function of the adopted external radiation field. Its effect turns out to be quite interesting: a strong radiation field $\left(\chi>10^{3}\right)$ is needed to account for the large observed $\mathrm{CH}^{+}$column density. It is the enhanced photo-dissociation of $\mathrm{CH}_{2}^{+}$and $\mathrm{CH}_{3}^{+}$, both products of the rapid hydrogenation of $\mathrm{CH}^{+}$in the shock, that increases the abundance of $\mathrm{CH}^{+}$as $\chi$ increases. The shock model also predicts that both $\mathrm{CH}^{+}$and $\mathrm{H}_{2} \mathrm{O}$ are rather well distributed across the various velocity slices of the shock. This supports the assumption that the $\mathrm{CH}^{+}$and $\mathrm{H}_{2} \mathrm{O}$ blue line wings are similar, underlying the $\mathrm{CH}^{+}$line frequency determination.

\section{2. $\mathrm{CH}^{+}$absorption lines: turbulent dissipation models}

The large $\mathrm{CH}^{+}$column density observed in the foreground component corresponds to a los average of the $\mathrm{CH}^{+}$abundance, $\left[\mathrm{CH}^{+}\right] /[\mathrm{H}]_{\text {ave }}>1.2 \times 10^{-8}$, for $N(\mathrm{H})=1.4 \times$ $10^{22} \mathrm{~cm}^{-2}$ (see Sect. 2). It can be reproduced in the framework of TDR models. At any time, a very large number of tiny regions ( $l \sim 100 \mathrm{AU}$ ), altogether filling a small fraction of the entire los, are developing a transient warm chemistry triggered by dissipation bursts where local $\mathrm{CH}^{+}$abundances reach $10^{-6}$ (Godard et al. 2009). The filling factor of the ensemble of these tiny structures is set by the energy transfer rate in the turbulent cascade, $\epsilon=\rho v_{l}^{3} / l$, identified with the turbulent dissipation rate. The resulting average abundance is found to scale as $\left[\mathrm{CH}^{+}\right] /[\mathrm{H}]_{\mathrm{ave}}=$ $6.4 \times 10^{-9}\left(\epsilon / \epsilon_{0}\right)\left(n_{\mathrm{H}} / 50 \mathrm{~cm}^{-3}\right)^{-2.6}$ for an ambient radiation field $\chi=3$ in ISRF units, where $n_{\mathrm{H}}$ is the density of the gas in which the bursts occur and $\epsilon$ is a non-local quantity of galactic average $\epsilon_{0}=2 \times 10^{-25} \mathrm{erg} \mathrm{cm}^{-3} \mathrm{~s}^{-1}$ (see Godard et al. 2009). Fluctuations by two orders of magnitude are observed in the ISM about that galactic average, the highest values being reached in active star-forming regions, such as Cygnus X. We thus expect $\epsilon$ to be up to 100 times higher than average in Cygnus X, so that the observed $\mathrm{CH}^{+}$abundance can be produced by intermittent dissipation of turbulence occurring in gas of density up to $\sim 250 \mathrm{~cm}^{-3}$. The total number of tiny structures along that $1.7 \mathrm{kpc} l o s$ is found to scale as $\sim 3.5 \times 10^{3}\left(\epsilon / \epsilon_{0}\right)\left(n_{\mathrm{H}} / 50 \mathrm{~cm}^{-3}\right)^{-2}$.

\section{Conclusion}

We have detected the line profile of $\mathrm{CH}^{+}(1-0)$ towards the DR 21 massive star-forming region with the HIFI instrument, obtaining several major results. The line is a combination of broad emission and almost saturated broad absorption, which have comparable integrated areas. The rest-frame frequency of the $\mathrm{CH}^{+} J=1-0$ line is inferred to be $v=835137 \pm 3 \mathrm{MHz}$. For the gas seen in emission, the $\mathrm{CH}^{+}$column density, of about $10^{13} \mathrm{~cm}^{-2}$, compares well to predictions of C-shock models propagating in dense, highly illuminated gas. Additional chemical modelling (and radiative transfer calculations) are needed to confirm the $\mathrm{C}$-shock framework and exploit the other line signatures. The large $\mathrm{CH}^{+}$column density in the foreground gas, $N\left(\mathrm{CH}^{+}\right) \sim 10^{14} \mathrm{~cm}^{-2}$, can be explained by the turbulent dissipation models in diffuse gas and confirms the large opacity of that line in the diffuse molecular gas.

Acknowledgements. HIFI has been designed and built by a consortium of institutes and university departments from across Europe, Canada and the United States (NASA) under the leadership of SRON, Netherlands Institute for Space Research, Groningen, The Netherlands, and with major contributions from Germany, France and the US. Consortium members are: Canada: CSA, U. Waterloo; France: CESR, LAB, LERMA, IRAM; Germany: KOSMA, MPIfR, MPS; Ireland: NUI Maynooth; Italy: ASI, IFSI-INAF, Osservatorio Astrofisico di Arcetri-INAF; Netherlands: SRON, TUD; Poland: CAMK, CBK; Spain: Observatorio Astronòmico Nacional (IGN), Centro de Astrobiologia; Sweden: Chalmers University of Technology - MC2, RSS \& GARD, Onsala Space Observatory, Swedish National Space Board, Stockholm University - Stockholm Observatory; Switzerland: ETH Zurich, FHNW; USA: CalTech, JPL, NHSC. M.G. and E.F. acknowledge the support from the Centre National de Recherche Spatiale (CNES). Part of this work was supported by the German DeutscheForschungsgemeinschaft, DFG project \# Os177/1-1. We thank G. Pineau des Forêts for providing us with his version of the code for C-shock models.

\section{References}

Agundez, M., Goicoechea, J. R., Cernicharo, J., et al. 2010, ApJ, 713, 662 Amano, T. 2010, ApJ, accepted

Cernicharo, J., Liu, X.-W., Gonzalez-Alfonso, E., et al. 1997, ApJ, 483, L65 Crawford, M. K., Genzel, R., Townes, C. H., et al. 1985, ApJ, 291, 755

Cruz-Gonzalez, I., Salas, L., \& Hiriart, D. 2007, Rev. Mex. Astron Astrofis, 43, 337

Cyganowski, C. J., Reid, M. J., Fish, V. L., \& Ho, P. T. P. 2003, ApJ, 596, 344

Davis, C. J., Kumar, M. S. N., Sandel, G., et al. 2007, MNRAS, 374, 29

Douglas, A. E., \& Herzberg, G. 1941, ApJ, 94, 381

Falgarone, E., Phillips, T. G., \& Pearson, J. C. 2005, ApJ, 634, L149

Flower, D. R., \& Pineau des Forêts, G. 1998, MNRAS, 297, 1182

Flower, D. R., \& Pineau des Forêts, G. 2003, MNRAS, 343, 390

Garden, R. P., \& Carlstrom, J. E. 1992, ApJ, 392, 602

Godard, B., Falgarone, E., \& Pineau des Forêts, G. 2009, A\&A, 495, 847

de Graauw, T., et al. 2010, A\&A, 518, L6

Hobbs, L. M., Thorburn, J. A., Oka, T., et al. 2004, ApJ, 615, 947

Jakob, H., Kramer, C., Simon, R., et al. 2007, A\&A, 461, 999

Kirby, L. 2009, ApJ, 694, 1056

Kobayashi, R., Koch, H., \& Meyer, D. N. 1993, Chem. Phys. Lett., 211, 94

Lesaffre, P., Gerin, M., \& Hennebelle, P. 2007, A\&A, 469, 949

Marshall, D. J., Robin, A. C., Reylé, C., et al. 2006, A\&A, 453, 635

Motte, F., Bontemps, S., Schilke, P., et al. 2007, A\&A, 476, 1243

Nadeau, D., \& Béland, S. 1988, AJ, 95, 136

Oliveira, C. M., Dupuis, J., Chayer, P., \& Moos, H. W. 2005, ApJ, 625, 232

Ossenkopf, V., Röllig, M., Simon, R., et al. 2010, A\&A, 518, L79

Pearson, J. C., \& Drouin, B. J. 2006, ApJ, 647, L83

Pilbratt, G. L., et al. 2010, A\&A, 518, L1

Pineau des Forêts, G., Flower, D. R., Hartquist, T. W., \& Dalgarno, A. 1986, MNRAS, 220, 801

Poglitsch, A., Herrmann, F., Genzel, R., et al. 1996, ApJ, 462, L43

Roberts, D. A., Dickel, H. R., \& Goss, W. M. 1997, ApJ, 476, 209

Roelfsema, P. R., Goss, W. M., \& Geballe, T. R. 1989, A\&A, 222, 247

Russell, A. P. G., Bally, J., Padman, R., \& Hills, R. E. 1992, ApJ, 387, 219

Schneider, N., Csengeri T., Bontemps, S., et al. 2010, A\&A, accepted [arXiv: 1003.4198]

Sternberg, A., \& Dalgarno, A. 1995, ApJS, 99, 565

van der Tak, F. F. S., Marseille, M. G., Herpin, F., et al. 2010, A\&A, 518, L107

Wilson, T. L., Gaume, R. A., Johnston, K. J., \& Tieftrunk, A. R. 1995, ApJ, 452, 693 
1 LERMA, CNRS, Observatoire de Paris \& Ecole Normale Supérieure, 24 rue Lhomond, 75005 Paris, France

e-mail: maryvonne.gerin@lra.ens.fr

2 I. Physikalisches Institut der Universität zu Köln, Zülpicher Straße 77, 50937 Köln, Germany

3 SRON Netherlands Institute for Space Research, PO Box 800, 9700 AV Groningen, The Netherlands

${ }^{4}$ Institut d'Astrophysique Spatiale, CNRS and Université Paris-Sud, Bât. 121, 91405 Orsay Cedex, France

5 Jet Propulsion Laboratory, Caltech, 4800 Oak Grove Drive, Pasadena CA 91109-8099, USA

${ }^{6}$ LERMA, CNRS \& Observatoire de Paris, 61 Av. de l'Observatoire, Paris, France

7 CESR, CNRS and Université Paul Sabatier, 9 Avenue du Colonel Roche, 31062 Toulouse, France

8 Institute for Astronomy, ETH Zürich, 8093 Zürich, Switzerland

9 Observatorio Astronómico Nacional (OAN), Apdo. 112, 28803 Alcalá de Henares (Madrid), Spain

10 Max-Planck-Institut für Radioastronomie, Auf dem Hügel 69, 53121 Bonn, Germany
11 Astronomy Department, University of Maryland, College Park, MD 20742, USA

12 Instituto de Radio Astronomía Milimétrica (IRAM), Avenida Divina Pastora 7, Local 20, 18012 Granada, Spain

13 IPAC/Caltech, MS 100-22, Pasadena, CA 91125, USA

14 Department of Astronomy and Astrophysics, University of Toronto, 60 St. George Street, Toronto, ON M5S 3H8, Canada

15 Centro de Astrobiología, CSIC-INTA, 28850 Madrid, Spain

16 Department of Physics and Astronomy, John Hopkins University, 3400 North Charles Street, Baltimore, MD 21218, USA

17 California Institute of Technology, 320-47, Pasadena, CA 911254700, USA

18 Kapteyn Astronomical Institute, University of Groningen, PO box 800, 9700 AV Groningen, The Netherlands

19 European Space Astronomy Centre, Urb. Villafranca del Castillo, PO Box 50727, Madrid 28080, Spain

20 University of Massachusetts, Astronomy Dept., Amherst, MA 01003-9305 USA

21 Department of Physics and Astronomy, University of Waterloo, N2L 3G1, Canada 